\title{
The optimal bid/ask spread in a Specialist System
}

\author{
Rosella Castellano \& Roy Cerqueti* \\ University of Macerata \\ Department of Economic and Financial Institutions \\ Via Crescimbeni, 20 - 62100 - Macerata, Italy \\ Tel.:+390733 2583246; Fax: +390733 2583205 \\ Email:\{castellano, roy.cerqueti\}@unimc.it
}

\begin{abstract}
In this work we propose a simple market model where some features of the Specialist System are analyzed. In particular, the specialist's obligation to display bid/ask quotes on the book within the bounds imposed by the Exchange is considered. The proposed model allows to analyze the effects of the specialist's interventions on the short term dynamics of bid/ask prices and address a relevant market design issue, that is determination and analysis of the optimal endogenous upper bound that - according to economic conditions - should be imposed by Stock Exchange on the quoted bid/ask spread. The institutional details are summarized in a few structural parameters and the focus is on the aggregate effects of excess demand/supply.
\end{abstract}

Keywords: Dynamic Optimization; Maximum Spread; Specialist; Regulated Brownian Motion.

\section{Introduction}

The architecture of Stock Exchanges represents a key issue which continues to receive great attention in theoretical and empirical literature, especially during time periods characterized by financial turmoil. Over the past fifteen years, research in this area has focused mainly on the impact of alternative trading systems on market quality and efficiency, since one of the most important factors characterizing the development of financial markets has been the proliferation of new markets and automated trading systems. Many automated markets use a pure order driven system characterized by a high degree of transparency, in which public limit orders are continuously displayed. Some other markets also offer the alternative of trading under a hybrid order driven system with a specialist and limit order book. Stocks traded in a hybrid order driven system are usually sampled on the

\footnotetext{
*Corresponding author
} 
basis of certain parameters subject to annual review (i.e. capitalization, average quoted bid/ask spread, daily average trading volume, turnover, market touch, floating supply, etc.) and ranked into bands of liquidity (Nimalemdran and Petrella, 2003, for a detailed description). Under this scheme, the specialist has the duty to continuously display ask and bid prices on the book and negotiate a minimum lot of shares daily. Maximum spread and minimum number of lots per day are commonly determined by some Stock Exchanges. The bid/ask quotes are posted by the specialist on the limit order book for being displayed on trading terminals to all market participants. Accordingly, specialist is not monopolistic in managing and displaying the book since any intermediary has access to electronic limit order book and can place limit orders that compete with the specialist's quotes. Specialists are generally rewarded for their market making services with a reduction on trading fees, bid/ask spread revenues and lower information costs due to a greater knowledge of the issuer company. In some instances, they may also receive a side payment from the issuer company and, if this is the case, the relationship between the specialist and the issuer is regulated by an agreement which can take two forms: the issuer company may provide some funds to specialist or, alternatively, share the specialist's profits (and losses) related to market making activity.

The analysis of the Specialist System is relevant for several reasons. First, the specialist continues to be the focal point of the order flow especially during time periods characterized by low liquidity and financial crisis. Specialists maintain a fair, competitive, orderly and efficient market, meaning that all orders have equal opportunities to interact and receive the best price. Even though specialists can never completely prevent a fall in prices, they have the chance to buy stocks at different prices to fill in gaps and make the transition from one price to another more orderly. This cushioning process provides buyers and sellers with a better opportunity to enter the market and, eventually, restore the equilibrium (Nimalendran and Petrella, 2003; Heidle and Huang, 2002). Second, adopting a Walrasian approach, specialist's intervention may contribute to explain price discovery processes of actively traded stocks. In particular, by optimizing the spread, the specialist controls the imbalances arising from excess demand/supply and participates to processes that lead markets toward equilibrium.

In analogy with several previous empirical and theoretical studies, the focus of this paper is on the analysis of the Specialist System for markets in which stocks are actively traded (Anand and Weaver, 2006; Corwin, 2004; Parlour and Seppi, 2003; Fernando, 2003; Bondarenko and Sung, 2003; Madhavan, 2000; just to name a few of them), and on market makers that actively adjust the spread in response to fluctuations in the excess demand/supply (see amongst others: Stoll 1978; Madhavan and Smidt, 1993).

In this work, some features of the Specialist System are modeled. In line with the literature which relates to the microstructure of price processes, we examine the effects of the specialist's trading behavior, taking into account her/his obligation to display on the book the bid/ask quotes that do 
not differ for a percentage (spread) higher than that established by the Exchange, i.e. maximum allowed spread. In other words, within the framework of a simple market model, we aim at analyzing the primary function of the specialist, that is her/his commitment to provide liquidity to the market and, consequently, quote bid/ask prices in accordance with the maximum allowed spread.

First, we set up a model with an exogenous maximum allowed spread. In particular, the setting of the endogenous optimal bid/ask quotes, in accordance with the maximum allowed spread fixed by the Exchange is determined, taking also into account stabilization costs. Secondly, we endogenize the maximum allowed spread by solving a dynamic optimization problem. Since spread dynamics are endogenous, we are able to derive a solution for the optimal maximum allowed spread and provide specific insights related with the effectiveness of the specialist's role. The solution of the model contributes to the literature on market architectures and highlights that the effectiveness of the Specialist's strategy is improved by a greater flexibility in the setting of the maximum allowed spread whose level should not be fixed a priori by the Regulator, but adapted to economic conditions and characteristics of the traded stock.

The paper is structured as follows: in section 2 the theoretical model is presented; section 3 reports the methodology followed to solve the dynamic optimization problem and optimize the bounds on the specialist's trading strategy; section 4 reports some numerical results and their implications in terms of market architecture; concluding remarks are presented in section 5; the Appendix reports some algebra.

\section{The economic framework}

In this section, a model for stock price dynamics which takes into account the specialist's interventions on bid/ask prices is presented. Here, the economic environment in which the specialist operates and her/his actions in the setting of the bid/ask quotes are explored.

In this respect, we propose a simple market framework where, refraining from modeling the various components of the market microstructure explicitly (i.e.: order arrivals, mechanism of order clearing, etc.), the institutional details are summarized in a few parameters and the focus is on the effects of the excess demand/supply on price changes.

We assume that trading on secondary market, at time $t$, takes place in a market where all trades clear at the same price subject to transaction costs. We follow the process of price formation suggested by Brennan and Subrahmanyam (1996) and Farmer and Joshi (2002), and assume that the infinitesimal transaction price change at time $t, d P_{t}$, is a linear function of the order flow $X_{t}$, also driven by a stochastic noise $\xi_{t}$ :

$$
d P_{t}=\lambda \cdot X_{t}+\xi_{t},
$$


where $\lambda>0$ represents the transaction cost parameter whose inverse can be regarded as a proxy for the factors determining the liquidity of the stock and a measure of market depth (i.e.: $\lambda$ parameterizes the liquidity continuum between the case in which the stock is frictionless, $\lambda=0$, and the case in which it is perfectly illiquid, $\lambda \rightarrow \infty)$. The stochastic term $\xi_{t}$ may represent the position taken in the market by noise traders, or liquidity traders, who submit orders at random; otherwise, it can simply represent some random perturbation in the price (Farmer and Joshi, 2002).

Define the bid/ask spread at time $t, S_{t}$, as:

$$
S_{t}=P_{t}^{a}-P_{t}^{b},
$$

where $P_{t}^{a}$ and $P_{t}^{b}$ are the bid and ask prices, respectively. Since the specialist has to promote an orderly transaction of prices, it is assumed:

$$
d P_{t}=\gamma S_{t},
$$

where $\gamma>0$ represents the percentage of the spread providing a change in the observed stock price. In this respect, equation (3) formalizes that the change in the trade price at time $t, d P_{t}$, linearly depends on the bid/ask spread which is controlled by the specialist for influencing the price of the transaction, cover the cost associated with its functions and yield a reasonable profit from her/his market making activity (Copeland and Galai, 1983; Glosten and Milgrom, 1985).

Denote by $Z_{t}$ the excess demand, with the convention that $Z_{t}>0$ represents positive excess demand and $Z_{t}<0$ positive excess supply. The excess demand is assumed to show a high correlation with order flows (Asparouhova et al., 2003, Bondarenko, 2001) and, at an intuitive level, this implies that, with decreasing order flow, demand becomes more elastic. Therefore, there exists a constant $\tilde{k}<0$ such that:

$$
Z_{t}=\tilde{k} \cdot X_{t} .
$$

By (1), (3) and (4), the excess demand becomes:

$$
Z_{t}=k \gamma S_{t}-k \xi_{t},
$$

where $k=\tilde{k} / \lambda$.

Assuming the specialist stands ready to sell $Q_{t}^{a}$, for partially or completely offsetting an excess demand, and to buy $Q_{t}^{b}$ in case of excess supply, the transaction size at time $t$ is defined as:

$$
Q_{t}-\bar{Q}_{t}=\left\{\begin{array}{c}
Q_{t}^{a}>0 \\
Q_{t}^{b}<0,
\end{array}\right.
$$

where $\bar{Q}_{t}$ is the quantity at which normal demand and supply are balanced.

In this framework, it is assumed that the specialist is equipped with a reserve fund, $I_{t}>0$, measured in equivalent units of the stock. The fund is provided by the issuer and it is used by the specialist 
for maintaining a fairly ordered and liquid market. Following Stoll (1978), we assume that short sales are not allowed, since the specialist should maintain a minimum capital in each stock to avoid extra costs due to short selling. As a consequence, the specialist is automatically suspended from her/his obligations when $I_{t} \leq 0$.

Let us now introduce the underlying value, or equilibrium price, of the stock at time $t$ and denote it as $P_{t}^{*}$. The specialist manages bid and ask prices also accordingly to the evolution of $P_{t}^{*}$ (Stoll, 1978, 1989; Goldman and Beja, 1979). Bid and ask prices are thus given by:

$$
\begin{aligned}
& P_{t}^{a}=\left[1+\frac{Q_{t}^{a}}{I_{t}}\right] P_{t}^{*}, \\
& P_{t}^{b}=\left[1+\frac{Q_{t}^{b}}{I_{t}}\right] P_{t}^{*},
\end{aligned}
$$

where $Q_{t}^{a},\left|Q_{t}^{b}\right|<I_{t}$.

Following Goldman and Beja (1979), the dynamics of $P_{t}^{*}$ are assumed to be stochastic:

$$
d P_{t}^{*}=P_{t}^{*}\left[\mu_{t} d t+\sigma_{t} d B_{t}\right]
$$

where $\mu_{t}$ is the instantaneous drift of the process, representing the expected rate of return at time $t ; \sigma_{t}^{2}$ is the instantaneous variance; $B_{t}$ is a standard Brownian Motion.

Applying Ito's Lemma to (6) and (7), given (8), the stochastic differentials for ask and bid prices are, respectively:

$$
\begin{aligned}
& d P_{t}^{a}=\left[1+\frac{Q_{t}^{a}}{I_{t}}\right] \cdot d P_{t}^{*}, \\
& d P_{t}^{b}=\left[1+\frac{Q_{t}^{b}}{I_{t}}\right] \cdot d P_{t}^{*} .
\end{aligned}
$$

By (9) and (10), the stochastic differential for the bid/ask spread, $d S$, is obtained:

$$
d S_{t}=\frac{Q_{t}^{a}-Q_{t}^{b}}{I_{t}} \cdot d P_{t}^{*} .
$$

The drift of the process describing the spread dynamics, given in (11), clearly depends also on the sign of $Q_{t}-\bar{Q}_{t}$.

If $Q_{t}-\bar{Q}_{t}=Q_{t}^{a}>0$ the specialist sells at a higher ask price to compensate for excess demand and, for a fixed bid price, this would increase the spread. On the contrary, if $Q_{t}-\bar{Q}_{t}=Q_{t}^{b}<0$, the specialist buys at a lower bid price to compensate for excess supply and this, for a fixed ask price, would increase the spread. The changes in the spread are inversely related to the value of the inventory, $I_{t}>0$. In other words, the specialist does not anticipate price changes, but reacts to the order flow by assuming the role of passive stabilizer in that she/he buys when prices fall and sells when prices go up. 


\subsection{The regulated bid-ask spread}

The focus of the specialist, as member of the Exchange, is to facilitate the trading of assigned stocks. Accordingly, her/his primary function could be summarized by the minimization of the excess demand defined in (5).

Due to the sign of $k$ in equation (5), the minimization of the excess demand, $Z_{t}$, implies the maximization of the endogenous process $S_{t}$ which can be intended as proxy for illiquidity and, at the same time, represents a source of profit for the specialist's market making services. The maximization of $S_{t}$ is therefore contradictory with respect to the role of the specialist, which is mainly to support liquidity as passive stabilizer. In order to avoid this mismatch, an upper bound on the spread, $S_{t}$, has been introduced in some Exchanges.

In this respect, we assume that the bid/ask spread, $S_{t}$, follows an Ito process regulated between two barriers, 0 and $\bar{S}$, which are the exogenous bounds imposed by Exchanges in the setting of bid/ask quotes. At $S=0$ a costless and rewardless infinitesimal regulator $d L$ is applied by the specialist to the bid/ask spread, $S$, pushing it upward. At $S=\bar{S}$, another regulator $d U$ is applied, taking $S$ instantaneously to a level $S^{\prime}<\bar{S}$. By (8) and (11), the stochastic differential equation for the regulated bid/ask spread, $S_{t}^{R}$, can be written as:

$$
d S_{t}^{R}=\frac{Q_{t}^{a}-Q_{t}^{b}}{I_{t}} \cdot\left[\mu_{t} P_{t}^{*} d t+\sigma_{t} P_{t}^{*} d B_{t}+d L_{t}-d U_{t}\right],
$$

where $d L_{t}$ and $d U_{t}$ are non negative and right-continuous, non decreasing stochastic processes ${ }^{1}$, applied by the specialist to keep the bid/ask spread within the exogenous bounds, $[0, \bar{S}]$. In particular, $U$ increases, $d U=\bar{S}-S^{\prime}>0$, only when $S=\bar{S} ; L$ is continuous and increases only when $S=0$.

Let the upper regulator $d U$ be operated at a stabilization cost $d C$, where $C$ is a right continuous, non negative, non decreasing process which increases only when $U$ does.

In order to endogenize the maximum allowed spread, we consider the specialist's utility maximization problem. The objective function or expected discounted performance of the $\left(\bar{S}, S^{\prime}\right)$-policy is defined as:

$$
\begin{gathered}
F_{\bar{S}, S^{\prime}}(S)=\mathbf{E}\left[\int_{0}^{+\infty} e^{-\delta t} u\left[Z\left(S_{t}\right)\right] d t-d C \mid S_{0}=S\right]= \\
=\mathbf{E}\left[\int_{0}^{+\infty} e^{-\delta t} u\left[k \gamma S_{t}-k \xi_{t}\right] d t-d C \mid S_{0}=S\right]
\end{gathered}
$$

where $S_{t}$ follows the regulated Brownian Motion given in $(12) ; u(\cdot)$ is the utility function introduced to assess the specialist's attitudes toward the trading behavior. Therefore, the value function is given by:

$$
H(S)=\sup _{S^{\prime}, \bar{S}} F_{\bar{S}, S^{\prime}}(S)
$$

Inside the tolerance band defined by the Exchange, $0<S<\bar{S}$, the control does not take place and the process $S$ moves of its own accord. As a consequence, the expected change in $F$ is due to the

\footnotetext{
${ }^{1}$ From now on, we will omit the superscript $R$.
} 
excess demand, $Z$, defined in (5), and effects of discounting.

The Hamiltonian of the problem defined by (5), (12) and (14) is ${ }^{2}$ :

$$
\delta F_{\bar{S}, S^{\prime}}(S)=u(k \gamma S-k \xi)+\frac{\mu P^{*}\left(Q^{a}-Q^{b}\right)}{I} \cdot \frac{\partial F_{\bar{S}, S^{\prime}}(S)}{\partial S}+\frac{\left(Q^{a}-Q^{b}\right)^{2} \sigma^{2}\left(P^{*}\right)^{2}}{2 I^{2}} \cdot \frac{\partial^{2} F_{\bar{S}, S^{\prime}}(S)}{\partial S^{2}} .
$$

The solution of (15) clearly depends on the choice of the utility function $u$. Standard differential equation theory gives that its general solution is obtained by adding to a particular solution $V(S)$, related to the term $u(k \gamma S-k \xi)$, the solutions associated to the homogeneous equation:

$$
F_{\bar{S}, S^{\prime}}(S)=V(S)+A_{1} \cdot e^{\alpha_{1} S}+A_{2} \cdot e^{\alpha_{2} S},
$$

with

$$
\alpha_{1,2}=\frac{-\frac{\mu P^{*}\left(Q^{a}-Q^{b}\right)}{I} \pm \sqrt{\frac{\mu^{2}\left(P^{*}\right)^{2}\left(Q^{a}-Q^{b}\right)^{2}}{I^{2}}+\frac{2 \delta \sigma^{2}\left(P^{*}\right)^{2}\left(Q^{a}-Q^{b}\right)^{2}}{I^{2}}}}{\frac{\sigma^{2}\left(P^{*}\right)^{2}\left(Q^{a}-Q^{b}\right)^{2}}{I^{2}}}
$$

and integration constants $A_{1}, A_{2} \in \mathbf{R}$.

\section{The optimal solution}

In the previous section we considered the case in which the maximum spread, $\bar{S}$, is determined by the Exchange to avoid a conflict between the private interest of the specialist and her/his social function related to stabilization of prices and liquidity.

In this section, since in the proposed model the dynamics of the spread are endogenous, we are able to provide also a solution for the optimal upper bound on the bid/ask spread (i.e.: the optimal maximum spread that an Exchange should impose in accordance with economic conditions and characteristics of the traded stock).

The trajectory of the associated process $F_{\bar{S}, S^{\prime}}$ cannot be discontinuous except when the regulator $d U$ is applied at $S=\bar{S}$. At that time, the behavior of $F$ depends on the stabilization costs, $C$. If $d C$ is finite, a jump $d F$ occurs with $d F=d C$. On the contrary, if the cost is infinitely small, no jump occurs. We hypothesize the existence of a positive number $\rho$ such that $C^{\prime}(0)=\rho$, i.e. the costs are increasing in 0 . Moreover, if some fixed costs exist, then $C(0)>0$; otherwise, $C(0)=0$. In the following either cases are considered.

\subsection{Finite and infinitesimal costs}

Assume some fixed costs exist, $C(0)>0$. Next result shows the route to find the optimal $\bar{S}$ :

Theorem 1. Assume that $C(0)>0$. The optimal maximum allowed spread $\bar{S}$ is the solution of the following second-order ordinary differential equation:

$$
\left\{V^{\prime}(0) \alpha_{1} e^{\alpha_{1} \bar{S}}-V^{\prime \prime}(\bar{S})\right\} \cdot\left(e^{\alpha_{2} \bar{S}}-e^{\alpha_{1} \bar{S}}\right)--\left\{V^{\prime}(0) e^{\alpha_{1} \bar{S}}-V^{\prime}(\bar{S})-\rho\right\} \cdot\left(\alpha_{2} e^{\alpha_{2} \bar{S}}-\alpha_{1} e^{\alpha_{1} \bar{S}}\right)=0 .
$$

\footnotetext{
${ }^{2}$ The subscripts indicating time dependence are omitted since we refer to the initial values of the variables.
} 
The proof is reported in the Appendix.

Here we also consider the limit situation characterized by absence of fixed costs for the control of spread dynamics, $d S_{t}$. Assume that the regulator $d U$ is of small magnitude and that stabilization costs have a null fixed component, $C(0)=0$, with infinitesimal marginal cost given by stabilization policy, $C^{\prime}(0)=\rho$. In this case, the presence of infinitesimal costs implies that infinitesimal moves are optimal. The optimization of $A_{1}$ and $A_{2}$ is not sufficient to obtain the optimal threshold, $\bar{S}$, and it is necessary to introduce the so-called super contact condition (Dumas, 1991), involving the second derivative of the objective function, $F$. The following result holds:

Theorem 2. Assume that $C(0)=0$. The optimal maximum allowed spread $\bar{S}$ solves the following second-order ordinary differential equation:

$$
V^{\prime \prime}(\bar{S})+\left[-\frac{V^{\prime}(0) e^{\alpha_{1} \bar{S}}-V^{\prime}(\bar{S})-\rho}{e^{\alpha_{2} \bar{S}}-e^{\alpha_{1} \bar{S}}}-V^{\prime}(0)\right] \cdot \alpha_{1} e^{\alpha_{1} \bar{S}}+\left[\frac{V^{\prime}(0) e^{\alpha_{1} \bar{S}}-V^{\prime}(\bar{S})-\rho}{e^{\alpha_{2} \bar{S}}-e^{\alpha_{1} \bar{S}}}\right] \cdot \alpha_{2} e^{\alpha_{2} \bar{S}}=0
$$

The proof is presented in the Appendix.

\subsection{The optimal maximum spread in a risk-neutral setting}

The proposed model is very general, since it can be solved for different specialist's attitudes toward risk. Hereinafter, following the literature (i.e. O'Hara, 1995, just to give a representative reference), the specialist is assumed to be risk-neutral. With this respect, the search for the optimal maximum allowed spread (i.e. the optimizing threshold) is solved using a linear utility function, $u(x)=x$, whereas both cases of finite and infinitesimal costs are considered.

When $C(0)>0$, equation (15) becomes:

$$
\delta F=k \gamma S-k \xi+\frac{\partial F}{\partial S} \cdot \frac{\left(Q^{a}-Q^{b}\right) \mu P^{*}}{I}+\frac{1}{2} \frac{\partial^{2} F}{\partial S^{2}} \cdot \frac{\left(Q^{a}-Q^{b}\right)^{2} \sigma^{2}\left(P^{*}\right)^{2}}{I^{2}} .
$$

A particular solution of (20) is:

$$
V(S)=\frac{k \gamma S}{\delta}+\frac{k}{\delta}\left[-\xi+\frac{\left(Q^{a}-Q^{b}\right) \mu \gamma P^{*}}{\delta I}\right]
$$

and the optimal maximum allowed spread is given in implicit form by the smooth pasting conditions, formalized in the second equation of system (34) reported in the Appendix:

$$
\alpha_{2} e^{-\alpha_{1} \bar{S}}-\alpha_{1} e^{-\alpha_{2} \bar{S}}=\frac{k \gamma\left(\alpha_{2}-\alpha_{1}\right)}{\rho \delta+k \gamma} .
$$

If $C(0)=0,(19)$ coincides with (22).

\section{Some numerical results}

In this section we report and discuss some numerical results related to the sensitivity analysis of the optimal maximum allowed spread, derived from equation (22). Since the complexity of the problem 
does not allow to write explicitly the closed form solution of equation (22), a numerical study on the optimal maximum allowed spread, $\bar{S}$, is presented in order to better understand the results provided by the model.

In particular, the sensitivity of $\bar{S}$ with respect to marginal stabilization costs, $\rho$, discount factor, $\delta$ and volatility, $\sigma$, is analyzed.

To perform the analysis, a common set of parameters is defined as follows: $k=-0.5, P^{*}=10, \gamma=$ $.05, Q_{a}=0.1, Q_{b}=0, \mu=0.05, S=0.03, I=1.3$.

- Sensitivity of the optimal maximum allowed spread, $\bar{S}$, with respect to stabilization costs, $\rho$. In this case we set $\delta=0.05, \sigma=0.02$ and $\rho \in[0.01,0.04]$, since stabilization costs usually may range from one to four per cent, depending on a certain number of factors such as daily trading volume in the asset, size of the order, economic conditions and country in which the asset is traded. The curve of $\bar{S}$ depending on $\rho$, reported in Figure 1, shows that the optimal maximum allowed spread increases with stabilization costs. This result is perfectly in line with the idea that the bid/ask spread is managed by the specialist also to cover the costs associated with her/his function of maintaining an orderly and liquid market and implies that, in presence of increasing stabilization costs, the Exchange should set the maximum spread at a higher levels, in order to provide the specialist with more flexibility.

\section{INSERT FIGURE 1 ABOUT HERE}

- Sensitivity of optimal maximum allowed spread, $\bar{S}$, with respect to discount factor, $\delta$.

Here we consider $\rho=0.03, \sigma=0.02$ and $\delta \in[0.01,0.05]$. The graph of $\bar{S}$ as function of the discount factor, $\delta$, displayed in Figure 2, points out that optimal maximum allowed spread grows with $\delta$. This finding can be interpreted as follows: as interest rates in the economy increase, the expected return required by investors tend to increase determining imbalances in the demand/supply which requires specialist's interventions on the bid/ask quotes and, hence, higher stabilization costs and spread. Again, the results of the model suggest that, in the presence of high interest rates, the Exchange should provide greater flexibility to the specialist, in order to enhance the effectiveness of her/his action.

\section{INSERT FIGURE 2 ABOUT HERE}

- Sensitivity of optimal maximum allowed spread, $\bar{S}$, with respect to volatility, $\sigma$.

In this case we consider $\rho=0.03, \delta=0.03$ and $\sigma \in[0.01,0.1]$. The optimal maximum spread as function of volatility, showed in Figure 3, highlights that $\bar{S}$ increases almost proportionally with $\sigma$. This is perfectly in line with empirical findings showing that the bid-ask spread increases with a greater price volatility in the traded asset (Copeland and Galai, 1983). At an intuitive level, this result can be explained by the fact that when the volatility of the traded stock 
increases the bid-ask spread is wider because the specialist may want to take more actions to stabilize price changes and ensure a more orderly transition in prices changes.

\section{INSERT FIGURE 3 ABOUT HERE}

\subsection{Market design implications}

The numerical results discussed in the previous section highlight that Exchanges should determine the maximum allowed spread in accordance with the conditions of the economic framework and peculiar characteristics of the traded stock. This greater flexibility in the setting of bid and ask quotes may guarantee a more effective specialist's action in the stabilization process, contributing to improve the social benefits deriving from an increasing liquidity in the traded asset. In particular, the results of the proposed model show that Exchanges should not fix a priori the maximum allowed spread, but adapt its level to contingent circumstances.

In this section we analyze the implications of the obtained results in a market architecture perspective.

First of all, it is worth to point out the twofold function of the maximum spread. By one hand, uninformed traders may be dissuaded from trading in the presence of widening spread, because potential gains deriving from trading activity could be significantly reduced. As a consequence, an increasing maximum allowed spread is associated to decreasing liquidity in the related stock, since the dissuasion effect on uninformed traders becomes stronger as the spread becomes wider.

On the other hand, a maximum spread rule may facilitate the process of price discovery. In this respect, it is worth noticing that a well established market architecture should facilitate transactions and establish, through trading and other market communications, the correct underlying value of an asset (i.e. the equilibrium price). The asset's underlying value is known, generally with potential noises, only to informed investors. As a consequence of this asymmetry, uninformed traders fluctuate randomly between buys and sell orders, while informed traders are clustered on the buy (sell) side if the asset is under (over)-priced in the market, pushing market prices towards the true values. Accordingly, a constraint on the spread, encouraging trading by both informed and uninformed investors, increases the speed of the price discovery process. More specifically, as the maximum allowed spread becomes narrower, the price discovery process becomes faster. At the same time, we must recall that the bid/ask spread represents also a reward for the market making services provided by the specialist.

The above highlights some of the reasons which have led some Exchanges to set the maximum allowed spread. On the other hand, the results of the proposed model emphasize the importance of determining the maximum allowed spread taking into account the contingent economic conditions and characteristics of the traded stock. As a result, stock markets should set the maximum allowed spread in order to balance the benefits deriving from a narrow spread, that encourages trading 
and improve liquidity, with a greater flexibility granted to the specialist in determining the optimal bid/ask spreads in presence of a maximum allowed level.

Moreover, the results of the sensitivity analysis reported in the previous section shows that $\bar{S}$ increases with increasing stabilization costs, $\rho$. Therefore, in order to increase the liquidity of the asset and not limit the specialist's stabilization action, the Exchange should implement architectures aiming at reducing stabilization costs in such a way that the specialist can tolerate a lower maximum allowed spread. In this respect, some incentives could be provided to the specialist, in order to facilitate her/his stabilization policy and make it less expensive.

The optimal maximum allowed spread increases also with respect to discount factor, $\delta$, since an increasing $\delta$ reduce also the specialist's expected utility. The discount factor, although outside the control of the Exchange, must be taken into close consideration in the process of endogenization of the maximum allowed spread that should not be fixed in such a way to discourage the specialist and make more difficult the achievement of her/his targets ${ }^{3}$.

Another result is related with the volatility in the traded stock, i.e. the endogenous threshold $\bar{S}$ increases with $\sigma$. This is a direct consequence of the fact that, as the riskiness of the asset increases, the duty of the market maker to improve liquidity becomes less easily accessible. Since the Exchange is not able to control the volatility of the listed products, an endogenous maximum allowed spread should be fixed according to the volatility of the traded asset, in order to facilitate the achievement of the specialist' primary function. This result is particularly suitable for Exchanges where thin stocks are traded.

\section{Concluding remarks}

In this paper, we propose an economic model describing the short term dynamics of stock prices and the effects of price stabilization policies due to the specialist's trading behavior. The focus is on the aggregate effects of excess demand/supply on price changes and the model is solved via dynamic optimization.

The model allows to derive endogenously the optimal dynamics for the bid/ask spread and the optimal bound that should be imposed on the spread by the Exchange according to economic conditions. Given the assumption of a risk neutral specialist, the model admits a unique optimal maximum bid/ask spread, regardless the presence of fixed and marginal costs in the regulator.

The sensitivity analysis performed on the optimal maximum spread shows that it should be set by the Exchange at a higher level in presence of increasing stabilization costs, discount factor and volatility in the traded asset. The resulting greater flexibility in the specialist action could make

\footnotetext{
${ }^{3}$ The Exchange should not fix the optimal bound, as commonly happens, only in accordance to parameters related with the liquidity rank of the asset. As our results show, the liquidity of the asset may be improved by considering also the conditions of the economic environment.
} 
more effective her/his efforts to stabilize prices and contribute to a more orderly and liquid market.

\section{References}

[1] Anand. A., Weaver D.G., 2006. The value of the specialist: empirical evidence from the CBOE. Journal of Financial Markets, 9, 100-118.

[2] Asparouhova, E., Bossaerts, P., Plott, C., 2003. Excess demand and equilibration in multisecurity financial markets: the empirical evidence. Journal of Financial Markets, 6, 1-21.

[3] Bondarenko O., 2001. Competing market makers,liquidity provision, and bid-ask spreads. Journal of Financial Markets, 4, 269-308.

[4] Bondarenko O., Sung J., 2003. Specialist participation and limit orders. Journal of Financial Markets, 6, 539-571.

[5] Brennan M,J., Subrahmanyam A., 1996. Market microstructure and asset pricing: on the compensation for illiquidity in stock returns. Journal of Financial Economics, 41, 441-464.

[6] Copeland T. C., Galai D., 1983. Information effects of the bid-ask spread. Journal of Finance, $38,1457-1469$.

[7] Corwin S.A., 2004. Specialist performance and new listing allocations on the NYSE: an empirical analysis. Journal of Financial Markets, 7, 27-51.

[8] Dixit A. K., 1991. A simplified treatment of the theory of optimal regulation of Brownian motion. Journal of Economic Dynamics and Control, 15, 657-673.

[9] Dumas B., 1991. Super contact and related optimality conditions. Journal of Economics Dynamic and Control, 15, 675-685.

[10] Farmer, J.D., Joshi, S., 2002. The price dynamics of common trading strategies. Journal of Economic Behavior and Organization, 49, 149-171.

[11] Fernando C. S., 2003. Commonality in liquidity: transmission of liquidity shocks across investors and securities. Journal of Financial Intermediation, 12, 233-254.

[12] Goldman M. B., Beja A., 1979. Market Prices vs. Equilibrium Prices: Returns' Variance, Serial Correlation, and the Role of the Specialist. The Journal of Finance, 34, 595-607.

[13] Glosten L., Milgrom P., 1985. Bid, Ask and transaction prices in a specialist market with heterogeneously informed traders. Journal of Financial Economics, 14, 71-100. 
[14] Harrison J.M., 1985. Brownian motion and stochastic flow systems, Wiley (New York, NY).

[15] Heidle, H., Huang, R.D., 2002. Information-based trading in dealer and auction markets: An analysis of exchange listings. Journal of Financial and Quantitative Analysis 37, 391-424.

[16] Madhavan A., 2000. Market microstructure: A survey, Journal of Financial Markets, 3, 205-258.

[17] Madhavan A., Smidt S., 1993. An analysis of changes in specialist inventories and quotations. The Journal of Finance, Vol. 48, 1595-1628.

[18] Nimalendran M., Petrella G., 2003. Do "thinly-traded" stocks benefit from specialist intervention?. Journal of Banking and Finance, 27, 1855-1857.

[19] O'Hara, M., 1995. Market Microstructure Theory. Blackwell Publishers, Cambridge, Massachusetts.

[20] Parlour C.A., Seppi D.J., 2003. Liquidity-Based Competition for Order Flow. The Review of Financial Studies, 16, 301-343.

[21] Shackleton M.B., Sodal S., 2005. Smooth pasting as rate of return equalization. Economics Letters 89, 200-206.

[22] Stoll H.R., 1989. Inferring the Components of the Bid-Ask Spread: Theory and Empirical Tests. The Journal of Finance, 44, 115-134.

[23] Stoll H.R., 1978. The supply of dealer services in securities markets, The Journal of Finance, $33,1133-1151$.

\section{Appendix}

\section{Proof of Theorem 1}

If $C(0)>0$, then it is possible to rewrite $F_{\bar{S}, S^{\prime}}(S)$ as follows:

$$
F_{\bar{S}, S^{\prime}}(\bar{S})=F_{\bar{S}, S^{\prime}}\left(S^{\prime}\right)-C\left(\bar{S}-S^{\prime}\right)=F_{\bar{S}, S^{\prime}}(\bar{S}-d U)-C(d U) .
$$

Since $d U$ is sufficiently small, we can approximate $F_{\bar{S}, S^{\prime}}(\bar{S})$ in $(23)$ as:

$$
F_{\bar{S}, S^{\prime}}(\bar{S})=F_{\bar{S}, S^{\prime}}(\bar{S})-\frac{\partial F_{\bar{S}, S^{\prime}}(\bar{S})}{\partial S} d U-C^{\prime}(0) d U .
$$

In this case, without any optimization on $\bar{S}$ and $S^{\prime}$ being involved, the value matching condition takes the form of a condition on the first derivative of the performance function $F$, (Shackleton and Sodal, 2005; Dumas, 1991; Dixit, 1991, Harrison, 1985). The identity in (24) implies that

$$
\frac{\partial F_{\bar{S}, S^{\prime}}(\bar{S})}{\partial S}=-C^{\prime}(0)=-\rho,
$$


and, for $S=0$, by definition of the process $d L$, it is:

$$
\frac{\partial F_{\bar{S}, S^{\prime}}(0)}{\partial S}=0 .
$$

Substituting the general solution, (16), into the boundary conditions, (25) and (26), we find the values for $A_{1}$ and $A_{2}$ which are, respectively:

$$
\begin{gathered}
A_{1}=-\frac{V^{\prime}(0) e^{\alpha_{1} \bar{S}}-V^{\prime}(\bar{S})-\rho}{\alpha_{1} \cdot\left(e^{\alpha_{2} \bar{S}}-e^{\alpha_{1} \bar{S}}\right)}-\frac{V^{\prime}(0)}{\alpha_{1}}, \\
A_{2}=\frac{V^{\prime}(0) e^{\alpha_{1} \bar{S}}-V^{\prime}(\bar{S})-\rho}{\alpha_{2} \cdot\left(e^{\alpha_{2} \bar{S}}-e^{\alpha_{1} \bar{S}}\right)}
\end{gathered}
$$

with $\alpha_{1}$ and $\alpha_{2}$ defined as in (17).

In order to endogenize the maximum allowed spread and determine its optimal value, the choices of $\bar{S}$ and $S^{\prime}$ must be optimized. The maximization of $A_{1}$ and $A_{2}$ is equivalent to maximize the performance $F_{\bar{S}, S^{\prime}}(S)$ with respect to $\bar{S}$ and $S^{\prime}$, i.e. to compute the value function $H(S)$. In other words, with the optimal choice of the upper bound, $\bar{S}$, the value of the performance index increases everywhere. Deriving $F_{\bar{S}, S^{\prime}}(\bar{S})$ of $(23)$ with respect to $\bar{S}$ and $S^{\prime}$, we have:

$$
\frac{\partial F_{\bar{S}, S^{\prime}}(\bar{S})}{\partial \bar{S}}=-\frac{\partial C\left(\bar{S}-S^{\prime}\right)}{\partial \bar{S}}
$$

and

$$
0=\frac{\partial F_{\bar{S}, S^{\prime}}\left(S^{\prime}\right)}{\partial S^{\prime}}+\frac{\partial C\left(\bar{S}-S^{\prime}\right)}{\partial S^{\prime}} .
$$

Assuming $\frac{\partial F_{\bar{S}, S^{\prime}}\left(\bar{S}-S^{\prime}\right)}{\partial S}=\frac{\partial C\left(\bar{S}-S^{\prime}\right)}{\partial S^{\prime}},(29)$ and (30) imply:

$$
\frac{\partial F_{\bar{S}, S^{\prime}}(\bar{S})}{\partial \bar{S}}=\frac{\partial F_{\bar{S}, S^{\prime}}\left(S^{\prime}\right)}{\partial S^{\prime}},
$$

where $F$ is given by (16) and the integration constants, $A_{1}=A_{1}(\bar{S})$ and $A_{2}=A_{2}(\bar{S})$, are defined as in (27) and (28).

After some algebra, condition (31) can be rewritten as:

$$
\begin{gathered}
V^{\prime}\left(S^{\prime}\right)+A_{1}\left(S^{\prime}\right) \alpha_{1} e^{\alpha_{1} S^{\prime}}+A_{2}\left(S^{\prime}\right) \alpha_{2} e^{\alpha_{2} S^{\prime}}= \\
=V^{\prime}(\bar{S})+A_{1}(\bar{S}) \alpha_{1} e^{\alpha_{1} \bar{S}}+A_{2}(\bar{S}) \alpha_{2} e^{\alpha_{2} \bar{S}}+\frac{\partial A_{1}(\bar{S})}{\partial \bar{S}} \cdot \alpha_{1} e^{\alpha_{1} \bar{S}}+\frac{\partial A_{2}(\bar{S})}{\partial \bar{S}} \cdot \alpha_{2} e^{\alpha_{2} \bar{S}} .
\end{gathered}
$$

The identity in (32) holds when:

$$
\left\{\begin{array}{c}
S^{\prime}=\bar{S} \\
\frac{\partial A_{1}(\bar{S})}{\partial \bar{S}}=0 \\
\frac{\partial A_{2}(\bar{S})}{\partial S}=0
\end{array}\right.
$$

with $A_{1}$ and $A_{2}$ defined as in (27-28).

The system (33) represents the first order (smooth pasting) condition to be used to determine the 
optimal values of $S^{\prime}$ and $\bar{S}$.

Since the first order derivatives of the integration constants depend on the choice of the utility function, the system (33) can be rewritten as follows:

$$
\left\{\begin{array}{c}
S^{\prime}=\bar{S} \\
\left\{V^{\prime}(0) \alpha_{1} e^{\alpha_{1} \bar{S}}-V^{\prime \prime}(\bar{S})\right\} \cdot\left(e^{\alpha_{2} \bar{S}}-e^{\alpha_{1} \bar{S}}\right)- \\
-\left\{V^{\prime}(0) e^{\alpha_{1} \bar{S}}-V^{\prime}(\bar{S})-\rho\right\} \cdot\left(\alpha_{2} e^{\alpha_{2} \bar{S}}-\alpha_{1} e^{\alpha_{1} \bar{S}}\right)=0 .
\end{array}\right.
$$

System (34) is exactly what we were searching for, and the result is proved.

\section{Proof of Theorem 2}

Assume that $C(0)=0$. The second derivative of $F$ is:

$$
\frac{\partial^{2} F_{\bar{S}, S^{\prime}}(\bar{S})}{\partial S^{2}}=0 .
$$

Integrating the smooth pasting condition, formalized in (25) and (26), with the super contact condition, given in (35), and substituting the explicit expression of the value function $F$ of (16), the following nonlinear system is obtained:

$$
\left\{\begin{array}{c}
V^{\prime}(\bar{S})+A_{1} \alpha_{1} \cdot e^{\alpha_{1} \bar{S}}+A_{2} \alpha_{2} \cdot e^{\alpha_{2} \bar{S}}=-\rho, \\
V^{\prime}(0)+A_{1} \alpha_{1}+A_{2} \alpha_{2}=0 \\
V^{\prime \prime}(\bar{S})+A_{1} \alpha_{1}^{2} \cdot e^{\alpha_{1} \bar{S}}+A_{2} \alpha_{2}^{2} \cdot e^{\alpha_{2} \bar{S}}=0 .
\end{array}\right.
$$

The solutions of system (36) are consistent with those obtained in the previous section. More precisely, since $A_{1}$ and $A_{2}$ have the same form specified in (27) and (28), substituting $A_{1}$ and $A_{2}$, given respectively in (27) and (28), into the third equation of (36), the optimal threshold for the spread, $\bar{S}$, as solution of the following nonlinear equation is obtained:

$$
V^{\prime \prime}(\bar{S})+\left[-\frac{V^{\prime}(0) e^{\alpha_{1} \bar{S}}-V^{\prime}(\bar{S})-\rho}{e^{\alpha_{2} \bar{S}}-e^{\alpha_{1} \bar{S}}}-V^{\prime}(0)\right] \cdot \alpha_{1} e^{\alpha_{1} \bar{S}}+\left[\frac{V^{\prime}(0) e^{\alpha_{1} \bar{S}}-V^{\prime}(\bar{S})-\rho}{e^{\alpha_{2} \bar{S}}-e^{\alpha_{1} \bar{S}}}\right] \cdot \alpha_{2} e^{\alpha_{2} \bar{S}}=0,
$$

and the Theorem is proved. 